\title{
The cerebellum in drug craving
}

Josep Moreno-Rius and Marta Miquel

Psychobiology, Universitat Jaume I, Castellon de la Plana, Spain

*Corresponding author: Marta Miquel Ph.D.

Psychobiology Area, Universitat Jaume I, Avenida Sos Baynat s/n. 12071 Castellón, Spain

Phone number: +34 696440177. miquel@uji.es

Conflict of Interest Statement: The authors of the present manuscript declare no conflict of interest.

Acknowledgments: This work was supported by grant UJI (14I307.01/1) 


\section{ABSTRACT}

Craving has been considered one of the core features of addiction. It can be defined as the urge or conscious desire to use a drug elicited by the drug itself, drug-associated cues or stressors. Craving plays a major role in relapse, even after prolonged periods of abstinence, as well as in the maintenance of drug seeking in non-abstinent addicts. The circuitry of craving includes medial parts of the prefrontal cortex, ventral striatal zones, ventral tegmental area, ventral pallidum, and limbic regions. Interestingly, the cerebellum shows reciprocal loops with many of these areas. The cerebellum has been linked traditionally to motor functions but increasing evidence indicates that this part of the brain is also involved in functions related to cognition, prediction, learning, and memory. Moreover, the functional neuroimaging studies that have addressed the study of craving in humans repeatedly demonstrate cerebellar activation when craving is elicited by the presentation of drug-related cues. However, the role of cerebellar activity in these craving episodes remains unknown. Therefore, the main goal of this review is to provide a brief update on craving studies and the traditional neural basis of this phenomenon, and then discuss and propose a hypothesis for the function of the cerebellum in craving episodes.

Keywords: Craving, cerebellum, memory, cue reactivity, prediction, expectations. 


\section{Introduction}

Craving is a very relevant concept in the addiction field as it has been hypothesized to underlie drug seeking and relapse in addicts (O'Brien et al., 1998). It has always referred to an urge or conscious desire to take a drug though such conceptualization has been a matter of debate. Indeed, there are some authors that consider that craving may also occur unconsciously (Miller \& Gold, 1994; Berridge and Robinson, 1995). Despite this disagreement, the importance of pavlovian associations with craving was recognized from the very beginning (Wikler, 1948). It is now accepted that drug-associated stimuli evoke drug memories, triggering craving and relapse (Robinson and Berridge, 1993; Robbins et al., 2008; Pickens et al., 2011)

Traditionally, craving has been associated with brain areas related to reward, motivation and memory, including prefrontal cortical areas (Grant et al., 1996); the striatum and ventral pallidum (Filbey et al., 2009; Wetherill et al., 2013); mesolimbic dopamine structures such as the ventral tegmental area (Goudriaan et al., 2013); and reward memory areas like the amygdala or the hippocampus (Kilts et al., 2001; Volkow et al., 2004).

Addiction literature has not paid much attention to the cerebellum until recently (Miquel et al., 2009, 2016; Moulton et al., 2014).The cerebellum is a hindbrain structure that contains more neurons than the rest of the brain (Herculano-Houzel, 2009). This structure presents some organizational similarities with the cerebral cortex (Herrup, 2000). During the last few decades, several findings have pointed to the possibility that other functions not linked to motor domains could require the cerebellum. Indeed, several studies have provided evidence of cerebellar involvement in some of the brain functions altered in addiction, such as memory 
(Sacchetti et al., 2004), prediction (Blakemore and Sirigu, 2003), and executive control (Bellebaum and Daum, 2007; see Miquel et al., 2016 for a recent review).

Animal studies show the cerebellum to be reciprocally interconnected with the brain areas traditionally related to addiction. As a matter of fact, the presence of dopaminergic synaptic components in the cerebellum of primates and rodents has been repeatedly demonstrated (Melchitzky and Lewis, 2000; Carbo-Gas et al., 2014a). This finding is consistent with the observed reciprocal connections between the cerebellum and the ventral tegmental area (VTA), the main source of dopamine in the mesocorticolimbic system (Ikai et al., 1992). Striatal projections to the cerebellum have also been documented by means of virus tracing studies (Bostan et al., 2010). Limbic zones also seem to be functionally connected to the cerebellum. For example, basolateral amygdala inactivation prevents learning-induced cerebellar LTP (Zhu et al., 2011). Moreover, it is clear that the cerebellum is functionally associated with the prefrontal cortex (Kelly and Strick, 2003), as well as other motor and associative cortices (see Bostan et al., 2013; D’Angelo and Casali, 2013 for a review). Animal findings regarding cerebellar-cortical and cerebellar-subcortical connections have been confirmed in humans by neuroimaging studies. Notably, functional magnetic resonance imaging studies, including resting-state and task-related functional connectivity confirm cerebellar relationships between the cortical and subcortical areas involved in addiction, and specifically in craving. Concretely, co-activations and functional connections between cerebellum and cortical structures, such as dorsolateral prefrontal cortex (Habas et al., 2009; Leutgeb et al., 2016; Moulton et al., 2011; Sang et al., 2012), orbitofrontal cortex (Addis et al., 2016; Habas et al., 2009; Leutgeb et al., 2016), anterior cingulate cortex (Addis et al., 2016; Moulton et al., 2011; Sang et al., 2012; Zeng et al., 2012), insula (Addis et al., 2016; Habas et al., 2009; Moulton et al., 2011; Sang et al, 2012), and inferior frontal gyrus (Addis et 
al., 2016; Moulton et al., 2011; Tomasi and Volkow, 2011) have been reported. Other subcortical structures such as amygdala (Leutgeb et al., 2016; Sang et al., 2012; Zeng et al., 2012), hippocampus (Onuki et al., 2015; Sang et al., 2012; Zeng et al., 2012), ventral tegmental area (Carnell et al., 2014; Etkin et al., 2009; Kline et al, 2016; Kwon et al., 2014), dorsal striatum (Moulton et al., 2011; Sang et al., 2012; Tomasi and Volkow, 2011), and ventral striatum (Cauda et al., 2011; Cservenka et al., 2014; Koehler et al., 2013) also have demonstrated to be connected to the cerebellum.

In addition, there is growing evidence that addictive drugs induce direct effects on cerebellar functioning and plasticity. As an example, alcohol modifies Purkinje neuron firing rates (Freund and Palmer, 1997); and chronic exposure to this drug increases AMPA-dependent calcium signalling in these cells (Netzeband et al., 1999). It is noteworthy that cerebellar degeneration is a common feature in long-term alcoholics and has been linked to the emotional and cognitive deficits that these patients suffer (Fitzpatrick et al., 2008). Psychostimulants also affect the cerebellum. It has been demonstrated that cocaine-induced sensitization has a big impact on cerebellar plasticity, altering the balance of plasticity-related proteins (Vazquez-Sanroman et al., 2015ab). The direction of plasticity changes depends on the length of the withdrawal period that precedes a new drug exposure (Vazquez-Sanroman et al., 2015ab). Also, cocaine-induced preference conditioning selectively increases c-FOS expression (an early transcription factor which acts as a marker of neuronal activity) in the cerebellar cortex (Carbo-Gas et al., 2014ab).

Numerous human neuroimaging studies have demonstrated activation in the cerebellum during the presentation of drug-associated cues (Anderson et al., 2006; Filbey et al., 2009; Grant et al., 1996). In almost every study, craving was elicited by the presentation of the cue. 
Nevertheless, in the absence of causal studies about the cerebellum's role in drug addiction, the functional significance of such cerebellar activation in craving episodes is unknown.

Given the aforementioned findings, the main goal of the present work is to review evidence about the involvement of the cerebellum in craving and to propose a hypothesis for its role. First, we present the current neurobiological model of craving. Then, we discuss cravingeliciting studies in which the activation of the cerebellum was shown. Finally, we suggest a working hypothesis to clarify the function cerebellar activation might play in the disturbing experience that craving assumes for an addict. We hope that the present review will help guide future experimental approaches to the subject.

\section{The circuitry of craving}

Research in both clinical and preclinical fields has provided knowledge about the brain areas and circuits that underlie the experience of craving. The reinstatement/relapse models have been the major contributors to elucidating the brain areas involved in animal craving. These models, which are able to elicit craving-like behaviors after the extinction of drug seeking in experimental animals, appear valid because the same stimuli that elicit craving-like behaviors in animals (Spanagel et al., 1998, Sanchis-Segura et al., 2006), also provoke craving in humans (Jaffe et al., 1989; Sinha et al., 1999; Grant et al., 1996). Basically, findings about the underlying circuitry parallel those derived from human research (see Bossert et al., 2013 for a recent review).

Brain correlates of human craving have been studied using the cue reactivity paradigm. Drug addicts are presented with drug-related cues under abstinence, while both craving levels and neurobiological parameters are evaluated (Carter and Tiffany, 1999). The frontal zones involved in craving seem to be the anterior cingulate cortex, orbitofrontal cortex, dorsolateral prefrontal cortex, and the inferior frontal gyrus (Grant et al., 1996; Bonson et al., 2002). The 
anterior cingulate cortex, a brain area related to self-control (Tang et al., 2015) and to rewardrelated cognitive processes (Shidara and Richmond, 2002) has been found to be active when drug-related cues are presented (Filbey et al., 2009; Myrick et al., 2004). Activity in the anterior cingulum seems to act as a predecessor of the onset of craving, being linked to the emotional response triggered by drug-related cues (Robbins et al., 2008; Wexler et al., 2001). Orbitofrontal cortex activity during craving episodes (Bonson et al., 2002) appears to be more related to salience attribution and expectation (Volkow and Baler, 2015). Also, consistent activation of the dorsolateral prefrontal cortex has been involved in the acquisition and storage of drug-conditioned memories, as well as in the formation of drug-related short-term working memory that drives behavior (Tang et al., 2015).

When addicts are exposed to drug-related cues, the inferior frontal gyrus is also activated (Grant et al., 1996; Tomasi et al., 2014). This region exerts an inhibitory effect on drug seeking (Goldstein and Volkow, 2011; Tang et al., 2015). Importantly, craving suppression was inversely correlated with the activity of this prefrontal area (Volkow et al., 2010). Moreover, gray matter in this area correlated with striatal D2/D3 receptor availability in methamphetamine users (Morales et al., 2015).

Subcortical activity during craving experience has been described in brain areas traditionally related to emotional memory and reward processing, including hippocampus (Volkow et al., 2004), amygdala (Kilts et al., 2001), and ventral striatum (Filbey et al., 2009). Dorsal striatal activity, which can be associated with habitual behavior and its maintenance (Everitt and Robbins, 2016), has been reported to predict cocaine craving (Volkow et al., 2006). Furthermore, the activation of the insula encodes a prediction of the dynamic representation of interoceptive drug effects (such as the bitter taste or the local anaesthesia induced by 
cocaine snorting) elicited by drug cues, which ultimately contributes partially to the conscious craving perception (Myrick et al., 2004; Naqvi and Bechara, 2009).

Interestingly, activation of the cerebellum was a common finding in the aforementioned imaging studies (Anderson et al., 2006; Bonson et al., 2002; Filbey et al., 2009; Grant et al., 1996; Kilts et al., 2001; Smolka et al., 2006; Tomasi et al., 2015). However, this region has been habitually overlooked probably because of the firmly established view of the cerebellum as a region exclusively committed to motor-related functions. Such highly ingrained views have hampered the conceptualization of this structure as a brain area with different functions from those classically described. In the forthcoming section, we will discuss these results in depth and suggest a working hypothesis for the role of the cerebellum during cue-induced craving.

\section{The cerebellum in craving}

The contemporary theories of addiction have raised the cardinal importance of drug-related conditioned cue memories to elicit conscious (craving) and unconscious (wanting) triggers of drug seeking (Robinson and Berridge, 1993). Interestingly, the vast majority of human imaging studies in which the cerebellum has been found to be active when craving is experienced were done by exposing addicts to drug-associated cues (Grant et al., 1996; Bonson et al., 2002; Kilts et al., 2001; Anderson et al., 2006; Filbey et al., 2009; Smolka et al., 2006; Tomasi et al., 2015). Similarly, animal research has demonstrated the selective activation of the cerebellum in rodents that express a preference towards cocaine-related cues (Carbo-Gas et al., 2014ab).

To the best of our knowledge, the first report that pointed to the cerebellum as a putative part of the circuitry underlying drug-dependent memories and craving was published by Grant and colleagues in 1996. This was a 18-fluorodeoxyglucose positron emission tomography (PET) 
study in which craving was elicited by presenting cocaine-related stimuli such as crack pipes, razorblades or actual cocaine plus a video in which the subject could see another person taking cocaine. Cerebellar activation when presenting the cocaine-related video and objects was prominently correlated with craving reports, although non-significant differences were found in such activation between cocaine abusers and control subjects. A subsequent study, which used a similar experimental setting but a PET scan with greater resolution, found significant differences in cerebellar activation in the posterior hemisphere, but no correlation with craving levels (Bonson et al., 2002). Nevertheless, another fMRI study (Risinger et al., 2005) showed again a correlation with craving scores, as well as with behavioral ratings of "high" and "rush". Drug craving correlated with activity in the vermis (cerebellar culmen), whereas self-reports of "high" and "rush" were positively correlated with overlapping vermis zones but negatively with hemisphere activity.

Regarding these data, one could think that cerebellar response might be more closely related to cocaine-induced positive affective states than to the motivation for drug seeking. Nevertheless, this conclusion was challenged by the substantial overlap in the cerebellum's pattern of activation observed when patients were presented with cocaine-associated cues and when the same patients were exposed to anger-related stimuli (Kilts et al., 2001). Such an overlap was observed in the left hemisphere. The cues used in this study were personalized scripts built from patients' reports of drug use and anger-related situations, which combined bodily sensations and the contextual details reported by the patients. Therefore, activity in the cerebellum during drug-related cue presentation cannot be exclusively linked to positive emotional states.

In a recent study of cocaine abusers, cocaine-related videos elicited broad cerebellar activation of the posterior cerebellum including vermis and cerebellar hemispheres. Such 
activation correlated with D2/D3 receptor availability in ventral and dorsal striatal zones (Tomasi et al., 2015), as well as with the valence attributed to the cues. These findings provide further evidence of functional relationships between the cerebellum and motivationrelated areas.

Cerebellar responses have been shown to be independent of either stimulus-modality or drug specificity. Neuroimaging findings showed that presenting cocaine addicts with visual or auditory cues resulted in greater activity in the anterior and posterior vermis (Anderson et al., 2006). In the same way, widespread activation of the vermis and cerebellar hemispheres was found when drug users were exposed to tactile marijuana cues (a pipe) (Filbey et al., 2009). Schneider and colleagues (2001) carried out a functional magnetic resonance imaging (fMRI) study in alcoholic patients and non-alcoholic matched controls in which they compared brain BOLD signals induced by an ethanol scent, before and after an antidepressant treatment. Imaging (fMRI) findings demonstrated greater activation of the vermis and hemispheres in alcoholics in response to the ethanol odor stimulation prior to the treatment. Olbrich et al. (2006) also used the smell of ethanol as a cue in a ${ }^{15} \mathrm{H}_{2} 0$ PET study of alcoholic patients. This study showed significantly higher cerebellar blood flow in both the vermis and left hemisphere in alcoholics. Moreover, it is not only ethanol odor that is able to elicit craving and cerebellar activity. Presenting ethanol-related pictures to adolescent binge drinkers also promoted both craving and bilateral cerebellar activation including the vermis (Brumback et al., 2015).

Similarly, the cerebellum of smokers showed higher levels of activation during the presentation of visual cues related to smoking paraphernalia or while watching images of other smokers (Smolka et al., 2006; McClernon et al., 2009). In this case, craving scores 
correlated with the activity of a specific region in the vermis. Moreover, cerebellar response was observed under abstinence, but not in a satiated condition (McClernon et al., 2009).

Additional evidence comes from opiate research. The presentation of heroin-related paraphernalia to addicts at different time points of withdrawal induced increased activity in the cerebellar vermis (Sell et al., 2000; Lou et al., 2012). Activation of the vermis was found in addicts that received methadone or abstinence-based treatment (Tabatabei-Jafari et al., 2014). Furthermore, cerebellar activity might be a biomarker for heroin relapse. Indeed, heroin relapsers showed higher levels of activity in the vermis, especially the posterior part, compared to non-relapsers when heroin-related cues were presented (Li et al., 2014).

Functional connectivity studies indicated increased connectivity between the cerebellum and several cortical zones in addicted patients presented with drug-related cues (Wang et al., 2013). However, impairment in functional connectivity between frontal cortex, midbrain, anterior cingulate cortex and the vermis (Tomasi et al., 2010) and between cerebellum and nucleus accumbens has also been described (Froeliger et al., 2015). Remarkably, Nacetylcysteine reversed such impairment and reduced craving scores (Froeliger et al., 2015). Additional studies suggest that the role of the cerebellum in processing reward-predicting cues seems to be shared with other compulsive-like disorders, such as binge eating disorder or obesity. Obese women showed higher functional connectivity between the midbrain/VTA and cerebellar hemispheres when they were exposed to highly caloric food cues (Carnell et al., 2014). In a recent study from the same lab, binge eaters exhibited greater functional connectivity between the cerebellum and dorsal ACC during the presentation of food-related cues (Geliebter et al., 2016). Intriguingly, there is an overlap in the fMRI signal evoked by cocaine and food-related cues in the cerebellum, which suggests a general role of this region in the processing of conditioned reinforcing cues in people suffering from compulsive-like 
disorders (Tomasi et al., 2015). It is feasible that brain reactivity to food cues in cocaine addicts could be mediated by the usual deficient nutritional conditions seen in these patients. Nevertheless, a recent fMRI study conducted in tobacco smokers also showed enhanced functional connectivity between the cerebellar vermis and the left supramarginal gyrus in smokers versus controls when they were presented with food cues (Garrison et al., 2016). Overall, these findings point to the cerebellum as part of the neural circuitry underlying abnormal reward reactivity in addicts or people with other types of compulsive-like disorders. Moreover, a recent meta-analysis (Noori et al., 2016) shows largely overlapping patterns of brain activation common to drug, food, sex and gambling cues including the cerebellum, which strongly supports the involvement of the cerebellum in motivation-related brain circuitry.

Other brain function related to craving and addiction in which the cerebellum has been Con formato: Fuente:Sin Negrita involved is salience. Salience could be described as the property of a stimulus that makes it differentially relevant from the others within an environment (Uddin, 2015). The attribution of salience to the surrounding environmental stimuli is an essential process for survival that allows individuals to perform adaptive behavioral responses to approach beneficial goals or avoid threatening ones (Borsook et al., 2013). Of note, one of the most influential addiction theories posits that such disease is driven by the attribution of excessive incentive salience to drugs and drug-associated cues (Robinson and Berridge, 1993). Data showing cerebellar activations when salient stimuli or cues are presented no matter their valence point to the involvement of this structure in such process (Moulton et al., 2011; Killgore et al., 2003; Anderson et al., 2006). Moreover, resting-state functional connectivity studies have allowed to unravel a functional network termed as the "salience network", which showed responsivity to salient events with independence of the sensory modality (Downar et al., 2002). Posterior 
studies have confirmed functional connections between the cerebellum and the core components of this network, such as insula, anterior cingulate cortex and temporoparietal junction_(Caulfield et al., 2015; Habas et al., 2009; Igelström et al., 2016; Moulton et al., 2011; Shinn et al., 2015).

\section{The cerebellum's role: craving or prediction?}

Overall, neuroimaging data have confirmed that the cerebellum is consistently activated when drug-associated cues are presented. However, there is no such consistency when correlational analysis between cerebellar activation and craving reports is performed. On the contrary, the available evidence does not point towards the cerebellum as being directly involved in the conscious experience of craving. Nevertheless, the possibility remains that several methodological factors could account for the variability found between studies. Variables such as the number of subjects, their age, gender, average amount of drug used, years of use, route of administration (especially in the case of cocaine) and duration of abstinence are not equivalent in the majority of studies. Differences in the neuroimaging techniques used, which include PET scans, fMRI scans and co-registrations of both images, might have also influenced the results. Despite this, drug-specific cerebellar activation does appear consistently, suggesting a role of the cerebellum in processing drug-related cues or in some cognitive process that takes place in parallel or very close in time to the conscious craving experience. In studies using visual cues, the vermis might be activated by smooth-pursuit eye movements (Tanabe et al., 2002). However, studies using other sensorial modalities also showed activation of the vermis (Schneider et al., 2001; Filbey et al., 2009). Moreover, the activation of cerebellar regions has been described with almost every addictive drug (Anderson et al., 2006; Lou et al., 2012; Filbey et al., 2009; Schneider et al., 2001; Smolka et 
al., 2006). Therefore, available evidence rules out the possibility that cue modality or drugspecific brain modifications cause the cerebellar response.

Expectation, a cognitive process that involves predictions of a particular future event or outcome before it occurs (Van de Meer and Redish, 2010), has been shown to act as a predictor of drug use (Stacy, 1995). It has also been proposed as being responsible for controlling craving and drug seeking elicited by drug-paired stimuli (Hogarth et al., 2007). Degrading expectations has been shown to be an effective method to extinguish cue-induced drug seeking in addicts (Hogarth et al., 2014). Remarkably, significant activation of the cerebellum was observed when subjects were faced with a cocaine-related interview that included recall of previous drug experiences (Wang et al., 1999). In the control interview, participants were asked to describe their genogram. These results shed light on the cerebellar role in drug-related memories given that although the control interview also required memory retrieval no significant changes in cerebellar activity were demonstrated. Moreover, the expected administration of methylphenidate evoked greater activation of the vermis and lateral cerebellum than unexpected administration (Volkow et al., 2003). When expected versus unexpected drug treatments were compared, a significant response was still found in the vermis and the thalamus, but the increase in the vermis could not be related to the reinforcing effects. Again, these findings point to a role for the cerebellum in prediction more than in the reward experience linked to drug administration.

Therefore, a plausible explanation for the cerebellar response during cue-elicited craving might be the previously identified role of the cerebellum in prediction (Carbo-Gas et al., 2014a; Miquel el al., 2016). This possibility is supported by theories on cerebellar function that involve this part of the brain in a generalized prediction process (Courchesne and Allen, 1997; D’Angelo and Casali, 2013). Relationships between expectations and prediction 
become evident when the former are defined as the latter. In fact, some authors consider expectations as one of the multiple prediction processes the brain accomplishes (Brown and Brüne, 2012). It is generally accepted that "forward controllers" perform prediction tasks. That is, brain circuits which are able to recruit forward models in order to accomplish these predictive functions. A forward model can be defined as an internal representation of the world that allows a subject to make predictions of the action outcomes (Van der Meer and Redish, 2010). The way it is supposed to work may consist of providing a representation of the functioning of a specific system in response to a particular environmental setting with specific demands using internal memory. Indeed, the cerebellum is thought to act as a forward controller (Ito, 2008). It is able to estimate the new state of the system regarding the environmental demands from the inputs of the current state. The predictive function of the cerebellum seems to be shared with several other brain cortical and subcortical areas, such as striatal zones (Tanaka et al., 2004), parietal lobes (Blakemore and Sirigu, 2003), and prefrontal cortices, especially the orbitofrontal cortex (Eppinger et al., 2015). The cerebellum would be in charge of fast, unconscious predictions, whereas cortical areas would be responsible for conscious, slower ones (Blakemore and Sirigu, 2003). Accordingly, the cerebellum would be involved in unconscious predictions of drug availability triggered by the presentation of the cue while monitoring the internal state under withdrawal. It would then create an anticipatory virtual scenario (D’Angelo and Casali, 2013) that would be crucial for selecting the appropriate response. In this case, cue exposure would trigger a cerebellumgenerated prediction of drug availability. This prediction will activate the preparation of the brain networks that are required to trigger drug seeking and taking behaviors. This explanation might account for the lack of consistent correlations found between conscious craving reports and cerebellar activation, given that this task would take place at a 
subconscious level. Furthermore, this working hypothesis might explain the specific cFos expression found in the cerebellum of rodents expressing a preference for a cocaine-paired odor cue that was not observed when the animal did not acquire this conditioned response (Carbo-Gas et al., 2014ab). A recent neuroimaging study that monitored brain activity during reward anticipation showed 4 active nodes encompassing broad cerebellar areas including the vermis (Jia et al., 2016). Nevertheless, the authors of the study did not propose a specific hypothesis for the cerebellar activity, as no correlation with performance in neuropsychological tests was found.

As we reported in the previous section, the activation of the cerebellum was not uniform, and Con formato: Fuente:Sin Negrita different patterns have been found. Indeed, very few studies have shown an involvement of the anterior cerebellum (Anderson et al., 2006; Filbey et al., 2009), whereas activation in the posterior cerebellum has been a common finding (Anderson et al., 2006; Bonson et al., 2002; Filbey et al., 2009; Kilts et al., 2001; Li et al., 2014; Olbrich et al., 2006; Schneider et al., 2001; Tomasi et al., 2015; Wang et al., 2013), even in the few studies that show activity in the anterior lobe. This is consistent with the sensory vs cognitive/affective dichotomy proposed for the cerebellar anterior-posterior lobes (Stoodley and Schmahmann, 2010). Also, with the theories that conceive craving as basically a cognitive phenomenon (Tiffany, 1999; Kozlowski and Wilkinson, 1987).

Within the posterior lobe, most studies report activation of the cerebellar vermis (Anderson et al., 2006; Filbey et al., 2009; Li et al., 2014; Schneider et al., 2001, Tomasi et al., 2015). This is consistent with the results coming from animal models, which show the cerebellar vermis is activated when animals are exposed to a drug-paired olfactory cue (Carbo-Gas et al., 2014ab). In fact, other non-drug rewards or their expectation, even aversive stimuli, have the capacity to evoke activity in the vermis (Casey et al., 2000; Martin-Solch et al., 2001; Miyagawa et al., 
2007; Rogers et al., 1999). Additionally, there are several reports which indicate that the cerebellar vermis is connected with the dopamine circuitry. Studies in rodents (Carbo-Gas et al, 2014a, Ikai et al., 1992), monkeys (Melchitzky and Lewis, 2000), and humans (Anderson et al., 2006) are indicative of dopaminergic synaptic components and transmission in the cerebellum across species. This evidence is accompanied by the fact that the cerebellar vermis has demonstrated to be linked to limbic and reward-related structures, such as amygdala (Sang et al., 2012; Yu et al., 2016), hippocampus (Sang et al., 2012), as well as ventral tegmental area (Rogers et al., 2011; Etkin et al., 2009; Kline et al, 2016; Kwon et al., 2014) and both dorsal (Tomasi and Volkow, 2011) ventral striatal zones (Cauda et al., 2011; Koehler et al., 2013). Accordingly, this part of the cerebellum could be able to integrate the incoming information from all these brain areas, which would encode the primary emotional and motivational aspects of the cue, to guide the response.

On the other hand, activity in the posterior cerebellar hemispheres has also been repeatedly found (Bonson et al., 2002; Filbey et al., 2009; Li et al., 2014; Risinger et al., 2005; Schneider et al., 2001; Tomasi et al., 2015). The cerebellar hemispheres seem to be involved in a wide variety of cognitive tasks, including language processing (Desmond et al., 1998; Frings et al., 2006; McDermott et al., 2003; Tieleman et al., 2005), working memory (Desmond et al., 1997; Salmi et al., 2010), and other executive functions (Schall et al., 2003; Blackwood et al., 2004). Remarkably, the posterior cerebellar hemispheres are functionally connected to different areas within the prefrontal cortices (Addis et al., 2016; Habas et al., 2009; O'Reilly et al., 2009). Moreover, like the prefrontal cortices, the lateral regions of cerebellar hemispheres are the most recent parts to evolve (Ito, 2008). Hence, it is likely that the most recently evolved parts of the brain are responsible for processing aspects related to specialized higher order cognitive functions involved in craving, such as interpretation of the cue, 
conscious memory processes or exerting control over craving itself or drug seeking behavior. In this case, and given that the different cerebellar areas are thought to perform a common computational function (D'Angelo and Casali, 2013), the cerebellar hemispheres could also provide a quick integration of the incoming, more cognitive information and, together with the integration of emotional and motivational aspects of the cue performed by the cerebellar vermis, would be able to provide the aforementioned prediction.

Still, this remains a hypothesis that needs to be tested and further studies are required to shed light on the issue. For instance, to the best of our knowledge there have been no animal experiments assessing the role of the cerebellum in cue- or drug-induced reinstatement, and thus reversible inactivation of cerebellar zones before the reinstatement test are recommended to provide causal evidence on the role of the cerebellum in the neurobiology of craving. To test the hypothesis that cerebellar activity reflects the functioning of a forward model that leads to predictions and expectations of drug availability, additional human imaging studies would be useful as well. Studies combining temporary modifications of cerebellar activity by means of transcranial magnetic stimulation together with masked and unmasked drug-related cue exposure would also be useful to assess the role of the cerebellum in these paradigms. Presentation of drug-related cues below the awareness threshold while monitoring cerebellar activity would also be useful to further verify this hypothesis. Similarly, an extinction experiment re-exposing subjects to the same cue setting repeatedly would allow the cerebellum's function in drug-induced memories to be elucidated. It is assumed that forward models are able to adapt to changing environmental demands (D'Angelo and Casali, 2013). Therefore, repeated exposure to the same cue setting without receiving the drug would affect the described activation pattern in the cerebellum. If this were the case, deactivation of the cerebellum should be found when not predicting the drug as available in that setting. It is 
possible, however, that cerebellar activity might persist with different activity patterns, given that some recent studies have pointed to a role for the cerebellum in extinction learning (Kattoor et al., 2014; Utz et al., 2015).

In conclusion, craving is a state that every addict has experienced and that contributes to the maintenance of drug seeking and taking behavior as well as to relapse. The cerebellar activation found in cue reactivity paradigms might involve prior processing of the conscious craving experience that implies the use of drug-induced cue memories to recruit forward models which lead to unconscious prediction of drug as available. This prediction would then lead to expectations of drug availability under the control of inferior medial prefrontal regions. Ultimately, they would be used to prepare the organism to perform the necessary actions to ensure drug taking. Characterization of the neural basis of this prediction process would also be helpful in order to develop strategies to block cue-induced expectation of drug availability by reducing the brain activation accompanying it, which may lead to a new therapeutic option to treat addictive disorders. 


\section{References}

Addis, D. R., Moloney, E. E., Tippett, L. J., Roberts, R., Hach, S. 2016. Characterizing cerebellar activity during autobiographical memory: ALE and functional connectivity investigations.

Neuropsychologia.

Anderson, C.M., Maas, L.C., Frederick, B. de B., Bendor, J.T., Spencer, T.J., Livni, E., Kaufman, M.J. 2006. Cerebellar vermis involvement in cocaine-related behaviors. Neuropsychopharmacol. $31(6), 1318-26$

Bellebaum, C., Daum, I. 2007. Cerebellar involvement in executive control. Cerebellum 6, 18492.

Berridge, K.C., Robinson, T.E. 1995. The mind of an addicted brain: neural sensitization of wanting versus liking. Curr. Dir. Psychol. Sci. 4(3) 71-6.

Blackwood, N., Simmons, A., Bentall, R., Murray, R., \& Howard, R. 2004. The cerebellum and decision making under uncertainty. Cogn. Brain Res. 20(1), 46-53.

Blakemore, S.J., Sirigu, A. 2003. Action prediction in the cerebellum and in the parietal lobe. Exp. Brain Res. 153, 239-45.

Bonson, K.R., Grant, S.J., Contoreggi, C.S., Links, J.M., Metcalfe, J., Weyl, H.L., London, E.D. 2002. Neural systems and cue-induced cocaine craving. Neuropsychopharmacol., 26(3), 376-86.

Borsook, D., Edwards, R., Elman, I., Becerra, L., Levine, J. 2013. Pain and analgesia: the value of salience circuits. Prog. Neurobiol. 104, 93-105.

Bossert, J.M., Marchant, N.J., Calu, D.J., Shaham, Y. 2013. The reinstatement model of drug relapse: Recent neurobiological findings, emerging research topics, and translational research. Psychopharmacology 229, 453-76.

Bostan, A.C., Dum, R.P., Strick, P.L. 2013. Cerebellar networks with the cerebral cortex and basal ganglia. Trends Cogn. Sci. 17, 241-54. 
Brown, E.C., Brüne, M. 2012. The role of prediction in social neuroscience. Front. Hum.

Neurosci. 6, 147.

Brumback, T., Squeglia, L.M., Jacobus, J., Pulido, C., Tapert, S.F., Brown, S.A. 2015. Adolescent heavy drinkers' amplified brain responses to alcohol cues decrease over one month of abstinence. Addict. Beh. 46, 45-52.

Carbo-Gas, M., Vazquez-Sanroman, D., Aguirre-Manzo, L., Coria-Avila, G.A., Manzo, J., Sanchis-Segura, C., Miquel, M. 2014a. Involving the cerebellum in cocaine-induced memory: pattern of cFos expression in mice trained to acquire conditioned preference for cocaine. Addict. Biol. 19(1), 61-76.

Carbo-Gas, M., Vazquez-Sanroman, D., Gil-Miravet, I., De Las Heras-Chanes, J., Coria-Avila, G.A., Manzo, J., Miquel, M. 2014b. Cerebellar hallmarks of conditioned preference for cocaine. Physiol. Beh. 132C, 24-35.

Carnell, S., Benson, L., Pantazatos, S.P., Hirsch, J., Geliebter, A. 2014. Amodal brain activation and functional connectivity in response to high-energy-density food cues in obesity. Obesity 22 , $2370-8$.

Carter, B.L., Tiffany, S.T. 1999. Meta-analysis of cue-reactivity in addiction research. Addiction 94(3), 327-40.

Casey, K. L., Svensson, P., Morrow, T. J., Raz, J., Jone, C., Minoshima, S. 2000. Selective opiate modulation of nociceptive processing in the human brain. J. Neurophysiol. 84(1), 525-533.

Cauda, F., Cavanna, A. E., D'agata, F., Sacco, K., Duca, S., Geminiani, G. C. 2011. Functional connectivity and coactivation of the nucleus accumbens: a combined functional connectivity and structure-based meta-analysis. J. Cogn. Neurosci. 23(10), 2864-77.

Caulfield, M. D., Zhu, D. C., McAuley, J. D., Servatius, R. J. 2015. Individual differences in resting-state functional connectivity with the executive network: support for a cerebellar role in anxiety vulnerability. Brain Struct. Funct. 1-13. 
Courchesne, E., Allen, G. 1997. Prediction and preparation, fundamental functions of the cerebellum. Learn. Mem. 4, 1-35.

Cservenka, A., Casimo, K., Fair, D. A., Nagel, B. J. 2014. Resting state functional connectivity of the nucleus accumbens in youth with a family history of alcoholism. Psychiatry Res.

Neuroimaging, 221(3), 210-9.

D’Angelo, E., Casali, S. 2013. Seeking a unified framework for cerebellar function and dysfunction: from circuit operations to cognition. Front. Neural Circuits, 6(January), Art 116.

Desmond, J. E., Gabrieli, J. D., Wagner, A. D., Ginier, B. L., Glover, G. H. 1997. Lobular patterns of cerebellar activation in verbal working-memory and finger-tapping tasks as revealed by functional MRI. J. Neurosci. 17(24), 9675-85.

Desmond, J. E., Gabrieli, J. D., Glover, G. H. 1998. Dissociation of frontal and cerebellar activity in a cognitive task: evidence for a distinction between selection and search. Neuroimage, 7(4), $368-76$.

Downar, J., Crawley, A. P., Mikulis, D. J., Davis, K. D. 2002. A cortical network sensitive to stimulus salience in a neutral behavioral context across multiple sensory modalities. J.

Neurophysiol. 87(1), 615-620.

Eppinger, B., Heekeren, H.R., Li, S.-C. 2015. Age-related prefrontal impairments implicate deficient prediction of future reward in older adults. Neurobiol. Aging 36, 2380-90.

Etkin, A., Prater, K. E., Schatzberg, A. F., Menon, V., Greicius, M. D. 2009. Disrupted amygdalar subregion functional connectivity and evidence of a compensatory network in generalized anxiety disorder. Arch. Gen Psychiatry, 66(12), 1361-72.

Everitt, B.J., Robbins, T.W. 2016. Drug addiction: Updating actions to habits to compulsions ten years on. Annu. Rev. Psychol. 67, 23-50

Filbey, F.M., Schacht, J.P., Myers, U.S., Chavez, R.S., Hutchison, K.E. 2009. Marijuana craving in the brain. PNAS 106(31), 13016-21. 
Fitzpatrick, L.E., Jackson, M., Crowe, S.F. 2008. The relationship between alcoholic cerebellar degeneration and cognitive and emotional functioning. Neurosci. Biobehav. Rev. 32(3), 466-85.

Freund, R.K., Palmer, M.R. 1997. Ethanol depression of cerebellar Purkinje neuron firing involves nicotinic acetylcholine receptors. Exp. Neurol. 143(2), 319-22.

Frings, M., Dimitrova, A., Schorn, C. F., Elles, H. G., Hein-Kropp, C., Gizewski, E. R., ... Timmann, D. 2006. Cerebellar involvement in verb generation: an fMRI study. Neurosci. Lett. 409(1), 19-23.

Froeliger, B., McConnell, P.A., Stankeviciute, N., McClure, E.A., Kalivas, P.W., Gray, K.M. 2015. The effects of $\mathrm{N}$-acetylcysteine on frontostriatal resting-state functional connectivity, withdrawal symptoms and smoking abstinence: A double-blind, placebo-controlled fMRI pilot study. Drug Alcohol Depend. 156, 234-42.

Garrison, K.A., Sinha, R., Lacadie, C.M., Scheinost, D., Jastreboff, A.M., Constable, R.T., Potenza, M.N. 2016. Functional connectivity during exposure to favorite-food, stress, and neutralrelaxing imagery differs between smokers and nonsmokers. Nic. Tob. Res.

Geliebter, A., Benson, L., Pantazatos, S.P., Hirsch, J., Carnell, S. 2016. Greater anterior cingulate activation and connectivity in response to visual and auditory high-calorie food cues in binge eating: Preliminary findings. Appetite 96, 195-202.

Goldstein, R. Z., Volkow, N. D. 2011. Dysfunction of the prefrontal cortex in addiction: neuroimaging findings and clinical implications. Nature Reviews Neuroscience, 12(11), 652-669. Goudriaan, A.E., Veltman, D.J., van den Brink, W., Dom, G., Schmaal, L. 2013.

Neurophysiological effects of modafinil on cue-exposure in cocaine dependence: A randomized placebo-controlled cross-over study using pharmacological fMRI. Addict. Behav. 38, 1509-17. Grant, S., London, E.D., Newlin, D.B., Villemagne, V.L., Liu, X., Contoreggi, C., Margolin, A. 1996. Activation of memory circuits during cue-elicited cocaine craving. PNAS 93(21), 12040-5. 
Habas, C., Kamdar, N., Nguyen, D., Prater, K., Beckmann, C. F., Menon, V., Greicius, M. D. 2009. Distinct cerebellar contributions to intrinsic connectivity networks. J Neurosci. 29(26), $8586-94$.

Herculano-Houzel, S. 2009. The human brain in numbers: a linearly scaled-up primate brain. Front. Hum. Neurosci. 3(November), article 31.

Herrup, K. 2000. Thoughts on the cerebellum as a model for cerebral cortical development and evolution. In Bock, G.R., Cardew, G. (Eds): Evolutionary Developmental Biology of the Cerebral Cortex. Chichester: Wiley.

Hogarth, L., Dickinson, A., Wright, A., Kouvaraki, M., Duka, T. 2007. The role of drug expectancy in the control of human drug seeking. Journal of Experimental Psychology: Anim. Behav. Processes, 33, 484-96.

Hogarth, L., Retzler, C., Munafò, M.R., Tran, D.M.D., Troisi, J.R., Rose, A.K., Jones, A., Field, M. 2014. Extinction of cue-evoked drug-seeking relies on degrading hierarchical instrumental expectancies. Behav. Res. Ther. 59, 61-70.

Igelström, K. M., Webb, T. W., \& Graziano, M. S. 2016. Functional Connectivity Between the Temporoparietal Cortex and Cerebellum in Autism Spectrum Disorder. Cereb. Cortex Igloi, K., Doeller, C.F., Paradis, A.-L., Benchenane, K., Berthoz, A., Burgess, N., Rondi-Reig, L. 2014. Interaction between hippocampus and cerebellum crus I in sequence-based but not placebased navigation. Cereb Cortex

Ikai, Y., Takada, M., Shinonaga, Y., Mizuno, N. 1992. Dopaminergic and non-dopaminergic neurons in the ventral tegmental area of the rat project, respectively, to the cerebellar cortex and deep cerebellar nuclei. Neuroscience 51(3), 719-28.

Ito, M. 2008. Control of mental activities by internal models in the cerebellum. Nat. Rev. Neurosci. 9, 304-13. 
Jaffe, J.H., Cascella, N.G., Kumor, K.M., Sherer, M.A., 1989. Cocaine-induced cocaine craving. Psychopharmacology 97, 59-64.

Jia, T., Macare, C., Desrivières, S., Gonzalez, D. A., Tao, C., Ji, X., ... Bokde, A. L. 2016. Neural basis of reward anticipation and its genetic determinants. PNAS, 113(14), 3879-3884.

Kattoor, J., Thürling, M., Gizewski, E.R., Forsting, M., Timmann, D., Elsenbruch, S. 2014.

Cerebellar contributions to different phases of visceral aversive extinction learning. Cerebellum 13(1), 1-8.

Kelly, R.M., Strick, P.L. 2003. Cerebellar loops with motor cortex and prefrontal cortex of a nonhuman primate. J. Neurosci. 23, 8432-44.

Killgore, W. D., Young, A. D., Femia, L. A., Bogorodzki, P., Rogowska, J., Yurgelun-Todd, D. A. 2003. Cortical and limbic activation during viewing of high-versus low-calorie foods. Neuroimage, 19(4), 1381-1394.

Kilts, C.D., Schweitzer, J.B., Quinn, C.K., Gross, R.E., Faber, T.L., Muhammad, F., Drexler, K.P. 2001. Neural activity related to drug craving in cocaine addiction. Arch. Gen. Psychiatry 58(4), $334-41$.

Kline, R. L., Zhang, S., Farr, O. M., Hu, S., Zaborszky, L., Samanez-Larkin, G. R., \& Li, C. S. R. 2016. The Effects of Methylphenidate on Resting-State Functional Connectivity of the Basal Nucleus of Meynert, Locus Coeruleus, and Ventral Tegmental Area in Healthy Adults. Front. Hum. Neurosci. 10.

Koehler, S., Ovadia-Caro, S., van der Meer, E., Villringer, A., Heinz, A., Romanczuk-Seiferth, N., Margulies, D. S. 2013. Increased functional connectivity between prefrontal cortex and reward system in pathological gambling. PLoS One, 8(12), e84565.

Kozlowski, L. T., \& Wilkinson, D. A. 1987. Use and misuse of the concept of craving by alcohol, tobacco, and drug researchers. Br. J. Addict. 82(1), 31-36. 
Kwon, H. G., \& Jang, S. H. 2014. Differences in neural connectivity between the substantia nigra and ventral tegmental area in the human brain. Front. Hum. Neurosci. 8, 41.

Leutgeb, V., Wabnegger, A., Leitner, M., Zussner, T., Scharmüller, W., Klug, D., Schienle, A.

(2016). Altered cerebellar-amygdala connectivity in violent offenders: A resting-state fMRI study. Neurosci. Lett. 610, 160-164.

Li, Q., Li, W., Wang, H., Wang, Y., Zhang, Y., Zhu, J., Zheng, Y., Zhang, D., Wang, L., Li, Y., Yan, X., Chang, H., Fan, M., Li, Z., Tian, J., Gold, M.S., Wang, W., Liu, Y. 2014. Predicting subsequent relapse by drug-related cue-induced brain activation in heroin addiction: an eventrelated functional magnetic resonance imaging study. Addict. Biol. 20(5), 968-978

Lou, M., Wang, E., Shen, Y., Wang, J. 2012. Cue-elicited craving in heroin addicts at different abstinent time: an fMRI pilot study. Subst. Use Misuse, 47(6), 631-9.

Martin-Sölch, C., Magyar, S., Künig, G., Missimer, J., Schultz, W., Leenders, K. 2001. Changes in brain activation associated with reward processing in smokers and nonsmokers. Experimental Brain Research, 139(3), 278-286.

McClernon, F.J., Kozink, R.V., Lutz, A. M., Rose, J.E. 2009. 24-h smoking abstinence potentiates fMRI-BOLD activation to smoking cues in cerebral cortex and dorsal striatum.

Psychopharmacology 204, 25-35.

Melchitzky, D.S., Lewis, D.A. 2000. Tyrosine hydroxylase- and dopamine transporterimmunoreactive axons in the primate cerebellum dopamine innervation. Neuropsychopharmacol. $22(5), 466-72$.

Miller, N.S., Gold, M.S., 1994. Dissociation of "conscious desire" (craving) from and relapse in alcohol and cocaine dependence. Ann. Clin. Psychiatry 6 (2), 99-106.

Miquel, M., Toledo, R., García, L.I., Coria-Avila, G.A., Manzo, J. 2009. Why should we keep the cerebellum in mind when thinking about addiction? Curr. Drug Ab. Rev. 2(1), 26-40. 
Miquel, M., Vazquez-Sanroman, D., Carbo-Gas, M., Gil-Miravet, I., Sanchis-Segura, C., Carulli, D., Manzo, J., Coria-Avila, G.A. 2016. Have we been ignoring the elephant in the room? Seven arguments for considering the cerebellum as part of addiction circuitry. Neurosci. Biobehav. Rev. $60,1-11$

Miyagawa, Y., Tsujimura, A., Fujita, K., Matsuoka, Y., Takahashi, T., Takao, T., ... Oku, N. 2007. Differential brain processing of audiovisual sexual stimuli in men: comparative positron emission tomography study of the initiation and maintenance of penile erection during sexual arousal.

Neuroimage, 36(3), 830-842.

Morales, A.M., Kohno, M., Robertson, C.L., Dean, A.C., Mandelkern, M.A., London, E.D. 2015.

Gray-matter volume, midbrain dopamine D2/D3 receptors and drug craving in methamphetamine users. Mol. Psychiatry 20, 764-71.

Moulton, E. A., Elman, I., Pendse, G., Schmahmann, J., Becerra, L., Borsook, D. 2011. Aversionrelated circuitry in the cerebellum: responses to noxious heat and unpleasant images. J. Neurosci. 31(10), 3795-804.

Moulton, E. A., Elman, I., Becerra, L. R., Goldstein, R. Z., \& Borsook, D. 2014. The cerebellum and addiction: insights gained from neuroimaging research. Addict. Biol. 19(3), 317-331.

Myrick, H., Anton, R.F., Li, X., Henderson, S., Drobes, D., Voronin, K., George, M.S. 2004.

Differential brain activity in alcoholics and social drinkers to alcohol cues: relationship to craving. Neuropsychopharmacol. 29(2), 393-402.

Naqvi, N. H., Bechara, A. 2009. The hidden island of addiction: the insula. Trends Neurosci. $32(1), 56-67$.

Netzeband, J.G., Trotter, C., Caguioa, J.N., Gruol, D.L. 1999. Chronic ethanol exposure enhances AMPA-elicited $\mathrm{Ca} 2+$ signals in the somatic and dendritic regions of cerebellar Purkinje neurons. Neurochem. Int. 35(2), 163-74. 
Noori, H. R., Linan, A. C., \& Spanagel, R. 2016. Largely overlapping neuronal substrates of reactivity to drug, gambling, food and sexual cues: A comprehensive meta-analysis. Eur.

Neuropsychopharmacol.

O'Brien, C.P., Childress, A.R., Ehrman, R, Robbins, S.J. 1998. Conditioning factors in drug abuse:

Can they explain compulsion? J. Psychopharmacol. 12(1), 15-22.

O'Reilly, J. X., Beckmann, C. F., Tomassini, V., Ramnani, N., \& Johansen-Berg, H. 2010. Distinct and overlapping functional zones in the cerebellum defined by resting state functional connectivity. Cereb. Cortex, 20(4), 953-65.

Olbrich, H.M., Valerius, G., Paris, C., Hagenbuch, F., Ebert, D., Juengling, F.D. 2006. Brain activation during craving for alcohol measured by positron emission tomography. N.Z. J.

Psychiatry 40, 171-8.

Onuki, Y., Van Someren, E. J., De Zeeuw, C. I., \& Van der Werf, Y. D. 2015. Hippocampalcerebellar interaction during spatio-temporal prediction. Cereb. Cortex 25(2), 313-21.

Pickens, C.L., Airavaara, M., Theberge, F., Fanous, S., Hope, B.T., Shaham, Y. 2011.

Neurobiology of the incubation of drug craving. Trends Neurosci. 34(8), 411-20.

Risinger, R.C., Salmeron, B.J., Ross, T.J., Amen, S.L., Sanfilipo, M., Hoffmann, R.G., Stein, E. 2005. Neural correlates of high and craving during cocaine self-administration using BOLD fMRI. NeuroImage 26(4), 1097-108.

Robbins, T.W., Ersche, K.D., Everitt, B.J. 2008. Drug addiction and the memory systems of the brain. Ann. NY Acad. Sci. 1141, 1-21.

Robinson, T. E., Berridge, K.C. 1993. The neural basis of drug craving: an incentive sensitization theory of addiction. Brain Res. Brain Res. Rev. 8, 247-91. 
Rogers, R. D., Owen, A. M., Middleton, H. C., Williams, E. J., Pickard, J. D., Sahakian, B. J., Robbins, T. W. 1999. Choosing between small, likely rewards and large, unlikely rewards activates inferior and orbital prefrontal cortex. J. Neurosci. 19(20), 9029-9038.

Rogers, T. D., Dickson, P. E., Heck, D. H., Goldowitz, D., Mittleman, G., Blaha, C. D. 2011. Connecting the dots of the cerebro- cerebellar role in cognitive function: Neuronal pathways for cerebellar modulation of dopamine release in the prefrontal cortex. Synapse, 65(11), 1204-1212. Sacchetti, B., Scelfo, B., Tempia, F., Strata, P. 2004. Long-term synaptic changes induced in the cerebellar cortex by fear conditioning. Neuron 42(6), 973-82.

Salmi, J., Pallesen, K. J., Neuvonen, T., Brattico, E., Korvenoja, A., Salonen, O., \& Carlson, S. (2010). Cognitive and motor loops of the human cerebro-cerebellar system. J. Cogn. Neurosci. $22(11), 2663-76$.

Sanchis-Segura, C., Borchardt, T., Vengeliene, V., Zghoul, T., Bachteler, D., Gass, P., Sprengel, R., Spanagel, R. 2006. Involvement of the AMPA receptor GluR-C subunit in alcohol-seeking behavior and relapse. J. Neurosci. 26, 1231-8.

Sang, L., Qin, W., Liu, Y., Han, W., Zhang, Y., Jiang, T., Yu, C. 2012. Resting-state functional connectivity of the vermal and hemispheric subregions of the cerebellum with both the cerebral cortical networks and subcortical structures. Neuroimage, 61(4), 1213-25.

Schall, U., Johnston, P., Lagopoulos, J., Jüptner, M., Jentzen, W., Thienel, R., ... Ward, P. B. 2003. Functional brain maps of Tower of London performance: a positron emission tomography and functional magnetic resonance imaging study. Neuroimage, 20(2), 1154-61.

Schneider, F., Habel, U., Wagner, M., Franke, P., Salloum, J.B., Shah, N.J., Zilles, K. 2001. Subcortical correlates of craving in recently abstinent alcoholic patients. Am. J. Psychiatry, 158(7), 1075-83. 

responses associated with cue evoked emotional states and heroin in opiate addicts. Drug Alcohol Depend. 60(2), 207-16

Shidara, M., Richmond, B.J. 2002. Anterior cingulate: Single neuronal signals related to degree of reward expectancy. Science (80). 296, 1709-11.

Shinn, A. K., Baker, J. T., Lewandowski, K. E., Öngür, D., Cohen, B. M. 2015. Aberrant cerebellar connectivity in motor and association networks in schizophrenia. Front. Hum. Neurosci. $9,134$.

Sinha, R., Catapano, D., O’Malley, S. 1999. Stress-induced craving and stress response in cocaine dependent individuals. Psychopharmacology 142, 343-51.

Smolka, M.N., Buhler, M., Klein, S., Zimmermann, U., Mann, K., Heinz, A. 2006. Severity of nicotine dependence modulates cue-induced brain activity in regions involved in motor preparation and imagery. Psychopharmacology 184, 577-88.

Spanagel, R., Sillaber, I., Zieglgänsberger, W., Corrigall, W.A., Stewart, J., Shaham, Y. 1998. Acamprosate suppresses the expression of morphine-induced sensitization in rats but does not affect heroin self-administration or relapse induced by heroin or stress. Psychopharmacology 139, $391-401$.

Stacy, A.W. 1995. Memory association and ambiguous cues in models of alcohol and marijuana use. Exp. Clin. Psychopharmacol. 3, 183-94.

Tabatabaei-Jafari, H., Ekhtiari, H., Ganjgahi, H., Hassani-Abharian, P., Oghabian, M.A., Moradi, A., Zarei, M. 2014. Patterns of brain activation during craving in heroin dependents successfully treated by methadone maintenance and abstinence-based treatments. J. Addict. Med. 8(2), 123-9. Tanabe, J., Tregellas, J., Miller, D., Ross, R.G., Freedman, R. 2002. Brain activation during smooth-pursuit eye movements. NeuroImage, 17(3), 1315-24. 
Tanaka, S.C., Doya, K., Okada, G., Ueda, K., Okamoto, Y., Yamawaki, S. 2004. Prediction of immediate and future rewards differentially recruits cortico-basal ganglia loops. Nat. Neurosci. 7, $887-93$

Tang, Y.-Y., Posner, M.I., Rothbart, M.K., Volkow, N.D. 2015. Circuitry of self-control and its role in reducing addiction. Trends. Cogn. Sci. 19, 439-44.

Tieleman, A., Seurinck, R., Deblaere, K., Vandemaele, P., Vingerhoets, G., Achten, E. 2005.

Stimulus pacing affects the activation of the medial temporal lobe during a semantic classification task: an fMRI study. Neuroimage, 26(2), 565-72.

Tiffany, S. T. 1999. Cognitive concepts of craving. Alcohol Res. Health, 23(3), 215-224.

Tomasi, D., Volkow, N.D., Wang, R., Carrillo, J.H., Maloney, T., Alia-Klein, N., Goldstein, R.Z. 2010. Disrupted functional connectivity with dopaminergic midbrain in cocaine abusers. PloS One, 5(5), e10815.

Tomasi, D., \& Volkow, N. D. 2011. Association between functional connectivity hubs and brain networks. Cereb. Cortex, 21(9), 2003-13.

Tomasi, D., Wang, G.-J., Wang, R., Caparelli, E.C., Logan, J., Volkow, N.D. 2015. Overlapping patterns of brain activation to food and cocaine cues in cocaine abusers: Association to striatal D2/D3 receptors. Hum. Brain Mapp. 36:120-36.

Uddin, L. Q. 2015. Salience processing and insular cortical function and dysfunction. Nat. Rev. Neurosci. 16(1), 55-61.

Utz, A., Thürling, M., Ernst, T.M., Hermann, A., Stark, R., Wolf, O.T., Timmann, D., Merz, C.J.

2015. Cerebellar vermis contributes to the extinction of conditioned fear. Neurosci. Lett. 604,

$173-7$.

Van der Meer, M.A., Redish, A.D. 2010. Expectancies in decision making, reinforcement learning, and ventral striatum. Front. Neurosci. 4, 29-37. 
Vazquez-Sanroman, D., Leto, K., Cerezo-Garcia, M., Carbo-Gas, M., Sanchis-Segura, C., Carulli, D., Rossi, F., Miquel, M. 2015a. The cerebellum on cocaine: plasticity and metaplasticity. Addict. Biol. 20(5), 941-955.

Vazquez-Sanroman, D., Carbo-Gas, M., Leto, K., Cerezo-Garcia, M., Gil-Miravet, I., SanchisSegura, C., Carulli, D., Rossi, F., Miquel, M. 2015b. Cocaine-induced plasticity in the cerebellum of sensitised mice. Psychopharmacology 232(24), 4455-4467.

Volkow, N.D., Baler, R.D. 2015. NOW vs LATER brain circuits: implications for obesity and addiction. Trends Neurosci. 38, 345-52.

Volkow, N.D., Fowler, J.S., Wang, G.-J. 2004. The addicted human brain viewed in the light of imaging studies: brain circuits and treatment strategies. Neuropharmacology 47 Suppl 1, 3-13. Volkow, N.D., Fowler, J.S., Wang, G.-J., Telang, F., Logan, J., Jayne, M., Ma, Y., Pradhan, K., Wong, C., Swanson, J.M. 2010. Cognitive control of drug craving inhibits brain reward regions in cocaine abusers. Neuroimage 49, 2536-43.

Volkow, N.D., Wang, G.-J., Ma, Y., Fowler, J.S., Zhu, W., Maynard, L., Swanson, J.M. 2003. Expectation enhances the regional brain metabolic and the reinforcing effects of stimulants in cocaine abusers. J. Neurosci. 23(36), 11461-8.

Volkow, N.D., Wang, G.-J., Telang, F., Fowler, J.S., Logan, J., Childress, A.-R., Jayne, M., Ma, Y., Wong, C. 2006. Cocaine cues and dopamine in dorsal striatum: mechanism of craving in cocaine addiction. J. Neurosci. 26, 6583-8.

Wang, G.-J., Volkow, N.D., Fowler, J.S., Cervany, P., Hitzemann, R.J., Pappas, N.R., Wang, C.F. 1999. Regional brain metabolic activation during craving elicited by recall of previous drug experiences. Life Sci. 64(9), 775-84.

Wang, Y., Zhu, J., Li, Q., Li, W., Wu, N., Zheng, Y., Wang, W. 2013. Altered fronto-striatal and fronto-cerebellar circuits in heroin-dependent individuals: a resting-state FMRI study. PLoS One, 8(3), e58098. 
Wetherill, R.R., Young, K.A., Jagannathan, K., Shin, J., O’Brien, C.P., Childress, A.R., Franklin, T.R. 2013. The impact of sex on brain responses to smoking cues: a perfusion fMRI study. Biol. Sex Differ. 4, 9

Wexler, B.E., Gottschalk, C.H., Fulbright, R.K., Prohovnik, I., Lacadie, C.M., Rounsaville, B.J., Gore, J.C. 2001. Functional magnetic resonance imaging of cocaine craving. Am. J. Psychiatry $158,86-95$

Wikler, A. 1948. Recent progress in research on the neurophysiologic basis of morphine addiction. Am. J. Psychiatry 105(5), 329-38.

Yu, X., Liu, L., Chen, W., Cao, Q., Zepf, F. D., Ji, G., ... Zang, Y. 2016. Integrity of Amygdala Subregion-Based Functional Networks and Emotional Lability in Drug-Naïve Boys With ADHD. J. Atten. Disord.

Zeng, L. L., Shen, H., Liu, L., Wang, L., Li, B., Fang, P., ... Hu, D. 2012. Identifying major depression using whole-brain functional connectivity: a multivariate pattern analysis. Brain 135(5), 1498-1507.

Zhu, L., Sacco, T., Strata, P., Sacchetti, B. 2011. Basolateral amygdala inactivation impairs learning-induced long-term potentiation in the cerebellar cortex. PLoS One 6, e16673. 